\title{
Fatores Associados à Prevalência de Sintomas Osteomusculares em Professores
}

\section{Factors associated with teachers' osteomuscular symptom prevalence}

\author{
Marcos Henrique Fernandes, Vera Maria da Rocha e \\ Angelo G. Roncalli da Costa-Oliveira
}

Universidade Federal do Rio Grande do Norte. marcoshenriquefernandes@bol.com.br,
vera.mrocha@ufrgs.br, roncalli@terra.com.br

Recebido em 7 Novembro 2008/Enviado para Modificação 15 Febrão 2009/Aprovado em 7 Marche 2009

RESUMO

Objetivo Determinar a prevalência de sintomas osteomusculares nos professores da rede municipal de Natal/RN, verificando a existência de associação entre as variáveis socioeconômicas, ocupacionais e de saúde com a presença dessa sintomatologia. Materiais e Métodos Trata-se de uma pesquisa descritiva com corte transversal, que teve como amostra 242 docentes da rede básica. Para a avaliação dos sintomas osteomusculares foi utilizado o questionário Nórdico. A análise estatística utilizou inicialmente procedimentos descritivos e o teste de Qui-Quadrado, e numa segunda etapa a análise de regresso logística multivariada, com nível de significância de $5 \%$. Resultados A prevalência da referida sintomatologia, considerando os últimos 12 meses, foi de $93 \%$ entre os investigados. Foi encontrada associação estatisticamente significativa entre o sexo feminino $(\mathrm{p}=0,021)$ e prática inadequada de atividade física $(p=0,037)$ com a presença de sintomas osteomusculares.

Conclusão A sintomatologia em questão pode estar pode estar comprometendo a qualidade de vida e o estado de saúde dos professores.

Palavras-Chave: Docentes, saúde do trabalhador, transtornos traumáticos cumulativos (fonte: DeCS, BIREME).

\section{ABSTRACT}

Objective Determining the prevalence of osteomuscular symptoms in public school teachers in Natal, Brazil, and verifying the existence of an association between socioeconomic, occupational and health variables and the presence of these symptoms.

Materials and Methods This was a descriptive cross-sectional study of a sample of 242 public elementary school teachers. The Nordic questionnaire was used for assessing osteomuscular symptoms. Statistical analysis initially involved descriptive procedures and the chi-square test; $5 \%$ significance level multivariate logistical regression analysis was used during a second stage. 
Results Symptom prevalence amongst the subjects investigated during the last 12 months was $93 \%$. A statistically significant association was found between being female $(p=0.021)$ and engaging in inadequate physical activity $(p=0.037)$ and the presence of osteomuscular symptoms.

Conclusion The symptoms revealed in this group may be compromising this group of workers' quality of life and health status.

Key Words: Teacher, workers' health, cumulative trauma disorder (source: $\mathrm{MeSH}$, NLM).

\section{RESUMEN}

Factores asociados a la prevalencia de síntomas osteomusculares en profesores

Objetivo Determinar la alta frecuencia de síntomas osteomusculares en los profesores de la red municipal de Natal/RN, y verificar la existencia de una asociación entre las variables socioeconómicas, ocupacionales y de salud con la presencia de esa sintomatología.

Materiales y Métodos Se trata de una investigación descriptiva con corte transversal, que tuvo como muestra 242 docentes de la red básica. Para la evaluación de los síntomas osteomusculares se utilizó el cuestionario Nórdico. El análisis utilizó procedimientos descriptivos y el test de Qui-Cuadrado inicialmente, y en una segunda etapa el análisis de regresión logística multivariada, con nivel significativo del $5 \%$.

Resultados La persistencia de la referida sintomatología, si consideramos los últimos 12 meses, fue del $93 \%$ entre los investigados. Se encontró una asociación estadísticamente significativa entre el sexo femenino $(p=0,021)$ y la práctica inadecuada de actividad física $(p=0,037)$ con la presencia de síntomas osteomusculares. Conclusión La sintomatología en cuestión puede estar comprometiendo la calidad de vida y el estado de salud de los profesores.

Palabras Clave: Docentes, salud del trabajador; trastornos de traumas acumulados (fuente: DeCS, BIREME).

$\mathrm{O}$ modelo de globalização levou ao processo de precarização do trabalho (1), tendo como consequiência direta o aumento das funções e da jornada das atividades profissionais, além da maior exposição a fatores de riscos para a saúde, afetando de maneira complexa o estilo de vida e o padrão de saúdedoença dos trabalhadores (2).

No Brasil uma das principais doenças relacionadas ao trabalho são os sintomas osteomusculares, também denominado de lesões por esforços repetitivos (LER) ou distúrbios osteomusculares relacionados ao trabalho (DORT). O referido quadro patológico afeta diversas categorias profissionais e apresenta uma considerável relevância social devido a sua abrangência e magnitude (3). É também responsável por grande parte dos gastos com tratamento de saúde e indenizações (4), sendo a segunda causa de afastamento do trabalho no Brasil (5). 
Os sintomas osteomusculares podem ser definidos como o auto relato de dor, formigamento ou dormência em nove diferentes regiões corporais, conforme o referencial teórico do questionário nórdico de avaliação de sintomatologia osteomuscular (6).

Dentre os fatores etiológicos, considerados de natureza multifatorial, destacamse os biomecânicos presentes na atividade de exigências repetitivas e desenvolvidas em ambientes planejados ergonomicamente inadequados, além das características individuais, do estilo de vida e das condições de trabalho (7). A maior prevalência da sintomatologia osteomuscular ocorre entre os trabalhadores jovens e do sexo feminino, que exercem atividades caracterizadas por grande esforço e repetitividade $(7,8)$.

Diversos fatores relacionados às condições e a organização do trabalho docente contribuem para surgimento de agravos à saúde e conseqüente comprometimento da qualidade de vida desses trabalhadores, como a desvalorização social, os baixos salários, a hierarquização e burocratização das relações de trabalho, além das deficiências de recursos humanos e logísticos. O atual ritmo acelerado de trabalho desenvolvido na escola pelos docentes tem obtido importante repercussão na área da saúde pública, devido ao aumento do adoecimento e afastamento desses profissionais (9).

Especificamente com relação ao acometimento por sintomas osteomusculares na população de professores, algumas pesquisas abordando essa temática foram realizadas no Brasil, sendo encontrados percentuais que variaram entre $40,9 \% \mathrm{e}$ $90,4 \%(10,11)$.

Nesse contexto, existem poucos estudos voltados para professores do ensino básico, que sofrem e adoecem em seu trabalho, e que não conseguem alternativas para melhoria das condições de trabalho, conformandose com o quadro desanimador em que se encontram (12). A problemática tornase acentuada quando levase em consideração estudos epidemiológicos sobre sintomas osteomusculares em trabalhadores docentes.

Dessa forma, a presente pesquisa objetivou determinar a prevalência de sintomas osteomusculares nos professores da rede municipal de Natal/RN, verificando a existência de associação entre as variáveis socioeconômicas (sexo, idade, estado civil, escolaridade, renda), ocupacionais (tempo de trabalho, carga horária, média de alunos por sala de aula, afastamento do trabalho no último ano 
e localidade do trabalho) e de saúde (atividade física e diagnóstico de doenças) com a presença da sintomatologia osteomuscular.

\section{MÉTODOS}

Este estudo se caracterizou como sendo descritivo-analítico e de corte transversal. A população foi constituída pelos professores da educação básica da rede municipal de Natal/RN, os quais segundo dados da Secretaria Municipal de Educação perfaziam um contingente de 2651 indivíduos no ano de 2007. Aamostra composta por 242 professores foi calculada tomando como base a população referida, um intervalo de confiança de $95 \%$ e margem de erro de $3 \%$.

Inicialmente foi determinado o número de indivíduos a serem selecionado em cada uma das quatro zonas distritais da cidade, respeitando a proporcionalidade entre o número total de docentes como o número amostral. Posteriormente os professores foram escolhidos de maneira aleatória através de sorteio simples, sendo excluídos aqueles que ocupavam cargos administrativos.

Após esclarecimento sobre os objetivos do estudo, foi entregue aos professores participantes, um questionário auto-administrável, composto por duas partes, cujo recolhimento foi realizado num momento posterior.

O primeiro bloco de questões correspondia a dados sócio-econômicos (sexo, idade, estado marital, renda e nível de escolaridade), dados ocupacionais (tempo de trabalho na docência, carga horária semanal de trabalho, e se a escola é localizada no mesmo bairro da residência do professor) e dados sobre a saúde que incluiu questões sobre doenças diagnosticadas e atividade física.

A questão sobre hábitos de atividade física foi adaptada do instrumento do perfil do estilo de vida individual (13), o qual foi validado na população brasileira (14). O professor podia atribuir a cada pergunta um valor de 0 (não faz parte do meu estilo de vida) a 3 (é verdadeira a informação no dia-a-dia). A partir da somatória da pontuação das questões os hábitos poderam ser classificado em adequados ( 6 a 9 pontos) e inadequados ( 0 a 3 pontos).

A segunda parte correspondia o questionário nórdico (Nordic questionnaire for the analysis of muscoloskeletal symptoms) para avaliação dos sintomas osteomusculares, o qual é muito utilizado devido a sua rápida eeconômica aplicação, sendo validado e adaptado culturalmente à língua portuguesa (15). 
O instrumento é composto por uma figura do corpo humano, mostrando as regiões anatômicas dos membros (pescoço, ombros, parte superior e inferior das costas, cotovelos, punhos/mãos, quadril/coxas, joelhos e tornozelos/pés). Nessa parte, o individuo responde com um X (xis) em cada questão, nas respostas " sim" ou "não", em relação à ocorrência de sintomas como dor, formigamento/dormência nos últimos 12 (doze) meses e nos últimos 7 (sete) dias.

Investiga, também, se os indivíduos procuraram auxílio de algum profissional de saúde e se foi impedido de realizar atividades nos últimos 12 (doze) meses, devido a estes sintomas.

O Projeto de Pesquisa foi aprovado pelo Comitê de Ética em Pesquisa da Universidade Federal do Rio Grande do Norte, obedecendo às normas da Resolução 196/ 96, incluindo o termo de consentimento livre e esclarecido.

A análise dos dados foi realizada através do programa SPSS-“Statistical Package for the Social Sciences" versão 15.0, sendo utilizada a estatística descritiva, com determinação das médias (x) e desvio-padrão (DP) para as variáveis quantitativas, e frequiências simples e relativas para as variáveis categóricas.

As relações entre as variáveis categóricas foram analisadas pelo teste de associação Qui-quadrado e exato de Fischer (para valores esperados menores que 5), sendo determinado o valor da OR (razão de chances) bruto com seus respectivos intervalos de confiança de $95 \%$. Em seguida foi utilizada a análise de regressão logística multivariada para investigar a existência de fatores de confusão, sendo levado em consideração associações entre variáveis independentes com p-valores inferiores a 0,2. A partir dessa análise foram obtidos valores da OR ajustados. O nível de significância adotado foi de $5 \%$.

\section{RESULTADOS}

Entre os professores investigados, $197(81,7 \%)$ eram do sexo feminino e 44 $(18,3 \%)$ do sexo masculino, tendo média de idade de 43,24 (DP \pm 9,23; IC a 95, 0 $\%=41,96-44,52$ ). Com relação ao estado marital, o maior número de docentes tinham união estável com 150 indivíduos (62\%), seguidos pelo grupo sem união estável com $64(26,4 \%)$.

Com relação à escolaridade, a maior parte 127 (53,4 \%) apresentava nível superior completo e $101(42,4 \%)$ pós-graduação. A renda média mensal dos 
docentes era de $\mathrm{R} \$ 1449,7$ ( $\mathrm{DP} \pm 642,60$; IC a $95 \%=1359,8-1539,5)$, correspondente a aproximadamente a quatro salários mínimos na época da coleta dos dados.

O tempo médio do exercício da atividade docente foi de 18 anos (DP $\pm 8,69$; IC a $95 \%=16,8-19,2)$. A carga horária semanal de trabalho apresentou média de $31,91$ horas ( $\mathrm{DP} \pm 14,82$; IC a $95 \%=29,8-33,9)$, e a média de alunos por sala de aula foi de 32,0 (DP $\pm 6,72$; IC a $95 \%=31,1-33,0)$. 64,9 \% dos professores não residiam no mesmo bairro em que estão localizadas as escolas que trabalham.

Com relação à atividade física, $46(19,3 \%)$ dos investigados apresentaram práticas consideradas adequadas e $192(80,7 \%)$ inadequadas. Amédia de doenças diagnosticada nos professores foi de 2,84 patologias $(\mathrm{DP} \pm 1,99$; IC a $95 \%=2,4-$ 2,9). Dentre essas, as que obtiveram maior percentual foram rinites/sinusites $(45,9 \%)$, varizes em membros inferiores $(44,2 \%)$, gastrite $(33,9 \%)$ e patologias nas cordas vocais $(21,5 \%)$.

A prevalência da sintomatologia osteomuscular no último ano, quando investigados, independente da região corporal afetada, foi de $93 \%$ entre os professores da rede municipal de ensino de Natal/RN. As regiões corporais em que se registraram mais queixas foram a parte superior das costas com $58,7 \%$, seguida pela parte inferior das costas com e pescoço com $53,7 \%$.

Do total de investigados, $47,7 \%$ responderam que a presença da sintomatologia osteomuscular nos últimos 12 meses impediu a realização de atividades da vida diária, tendo $51,5 \%$ dos participantes relatado ter realizado consulta a algum profissional da saúde devido ao referido problema.

A última pergunta do questionário Nórdico se referia ao acometimento dos sintomas nos últimos sete dias, o que foi respondido positivamente por $\quad 63,2 \%$ dos professores. Dentre as regiões onde foi apresentado um maior número de queixas, pode-se destacar a parte superior das costas com $28,5 \%$ e parte inferior das costas com $26,9 \%$. Todos os dados referentes ao questionário Nórdico encontram-se na Tabela 1.

Para análise das associações foi considerada apenas a presença de sintomas osteomusculares nos últimos 12 meses, independente da região afetada. Na análise univariada foi encontrada associação estatisticamente significante entre a sintomatologia investigada com o sexo feminino $(\mathrm{p}=0,004)$ e o diagnóstico de mais de 02 doenças $(\mathrm{p}=0,006)$. Após a análise multivariada, foi constatado que as 
variáveis sexo feminino $(\mathrm{p}=0,021)$ e prática inadequada de atividade física $(\mathrm{p}=0,037)$ apresentaram associação estatística significativa com a presença de sintomas osteomusculares nos últimos doze meses, conforme dados demonstrados na Tabela 2. A variável prática inadequada de atividade física ganhou significância nesta etapa da análise, sendo a mesma incluída devido ter obtido valor de $\mathrm{p}<0,2$ no teste do Qui-quadrado. O diagnóstico de mais de 02 doenças, após ajuste, perdeu a significância.

Tabela 1. Distribuição de sintomas osteomusculares, incapacidade funcional, procura por profissional da área de saúde entre professores da rede básica do município de Natal/RN

\begin{tabular}{|c|c|c|c|c|}
\hline Região Anatômica & $\begin{array}{c}\text { Sintomas } \\
\text { nos últimas } \\
12 \text { meses } \\
(\%)\end{array}$ & $\begin{array}{c}\text { Impedimento de realizar } \\
\text { atividades normais por } \\
\text { causa deste problema nos } \\
\text { últimos } 12 \text { meses } \\
(\%)\end{array}$ & $\begin{array}{c}\text { Consulta a algum } \\
\text { profissional da área da } \\
\text { saúde por causa desta } \\
\text { condição nos últimos } \\
12 \text { meses }(\%) \\
\end{array}$ & $\begin{array}{c}\text { Sintomas } \\
\text { nos } \\
\text { últimos } 7 \\
\text { dias } \\
(\%)\end{array}$ \\
\hline Pescoço & 53,7 & 17,4 & 20,7 & 24,0 \\
\hline Ombros & 50,4 & 16,5 & 17,8 & 26,4 \\
\hline $\begin{array}{l}\text { Parte superior das } \\
\text { costas }\end{array}$ & 58,7 & 15,3 & 17,8 & 28,5 \\
\hline Cotovelos & 11,6 & 4,5 & 4,5 & 5,0 \\
\hline Punhos/ mãos & 51,2 & 17,4 & 16,9 & 22,3 \\
\hline $\begin{array}{l}\text { Parte inferior das } \\
\text { costas }\end{array}$ & 53,7 & 22,7 & 18,6 & 26,9 \\
\hline Quadril/ coxas & 25,6 & 10,7 & 10,7 & 11,6 \\
\hline Joelhos & 38,4 & 15,7 & 14,9 & 18,2 \\
\hline Tornozelo/pé & 52,9 & 13,2 & 17,4 & 25,2 \\
\hline
\end{tabular}

Tabela 2. Análise multivariada para associação das variáveis Independentes com sintomas osteomusculares

\begin{tabular}{lcccc}
\hline Variável & $\begin{array}{c}\text { OR (IC 95\%) } \\
\text { Não ajustada }\end{array}$ & $p$ & $\begin{array}{c}\text { OR (IC 95\%) } \\
\text { Ajustada }\end{array}$ & $p$ \\
\hline Sexo Feminino* & $4,64(1,68-12,83)$ & 0,004 & $3,75(1,22-11,49)$ & 0,021 \\
$\begin{array}{l}\text { Atividade Física } \\
\text { inadequada* }\end{array}$ & $2,73(0,94-7,95)$ & 0,115 & $3,48(1,08-11,20)$ & 0,037 \\
\hline
\end{tabular}

* Fator associado à presença de sintomas osteomusculares

\section{DISCUSSÃO}

Diversos estudos realizados com amostras de professores do ensino básico apresentaram resultados sobre os dados sócio-econômicos e ocupacionais (carga horária, tempo de exercício da docência e o número de alunos por sala) semelhantes aos relatados na presente pesquisa, configurando um quadro homogêneo das características do trabalho docente no Brasil (11,16-19). 
Com relação específica à predominância de mulheres exercendo a prática da docência no ensino básico, pode ser explicada pelo processo histórico da entrada das mulheres no mercado de trabalho, onde grande parte delas ingressou no campo educacional, sendo a atividade docente rotulada como uma continuidade do trabalho doméstico, passando as professoras a assumir um papel de "mãe educadora" (20).

É importante ser ressaltado que a carga horária semanal relatada pelos investigados no presente estudo se refere apenas às atividades exercidas nas escolas, devendo ainda ser acrescentado o tempo para preparação de aulas, correções de atividades e tempo para o deslocamento ao local de trabalho, uma vez que a maior parte dos professores reside em bairros diferentes da localização da escola. A Lei de Diretrizes e Bases para a educação (Lei 9 394/96), no artigo 67 afirma que a carga horária para realização das atividades extra - classes deveriam estar inclusa no total de horas trabalhadas semanalmente (21).

Especificamente quando levado em consideração os últimos doze meses, a prevalência de $93 \%$ de sintomas osteomusculares nos indivíduos investigados no presente estudo, aponta para um elevado comprometimento na classe dos professores, o que também foi demonstrado nos resultados de outras pesquisas.

No estudo realizado com 212 docentes da rede pública de uma cidade do interior do estado de São Paulo, foi encontrada uma prevalência de 90,4 \% (22). Também foram pesquisadas as doenças ocupacionais diagnosticadas com maior frequiência nos atendimentos realizados a professores pelo Centro de Estudos da Saúde do Trabalhador da cidade de Salvador/BA, tendo os distúrbios osteomusculares acometidos a maior parte dos investigados (23). Na pesquisa realizada com 607 professores da rede municipal de Vitória/ES, 49,2 \% dos indivíduos relataram ter necessitado de atendimento médico em decorrência de problemas com transtornos osteoarticulares (17).

Os elevados valores de prevalência apontados anteriormente podem ser considerados como consequiência do novo paradigma do mundo do trabalho, que passou a obrigar o trabalhador a exercer suas atividades através de inadequados e intensos movimentos dos diversos segmentos corporais, provocando desordens músculo-tendinosas (24).

Nesse contexto, a prática da docência a longo prazo pode originar diversas patologias músculo-esqueléticas, muitas vezes relacionadas a quadros álgicos intensos, podendo acarretar em absenteísmos $(18,25)$. O referido quadro chama 
atenção devido à alta média de tempo de exercício profissional dos docentes da cidade de Natal/RN. Os referidos trabalhadores parecem que ainda não se adaptaram às mudanças exigidas pelo trabalho docente, levando ao surgimento de desequilíbrios na estrutura corporal e dor (25).

Características do trabalho docente como o esforço físico elevado, associados aos fatores biomecânicos presentes na atividade de exigências repetitivas e desenvolvidas em ambientes planejados ergonomicamente inadequados, são aspectos importantes que somados as características individuais do estilo de vida e das condições de trabalho, formam juntos uma rede interligadas de fatores que podem ajudar a explicar o aparecimento do referido quadro nos professores (9).

Tal problemática pode ser agravada, caso não seja devidamente diagnosticado e tratado os sintomas osteomusculares, o que poderá levar ao aparecimento de microlesões nos tendões e outras estruturas articulares, instituindo consequentemente um quadro crônico que pode resultar em uma situação mais grave de incapacidade funcional (25), onerando gastos com tratamento de saúde e indenizações (4).

No presente estudo, os fatores apontados na análise multivariada que estão associados à presença de sintomas osteomusculares nos professores investigados, foram o sexo feminino e a prática inadequada de atividade física.

Especificamente com relação à variável sexo feminino, tal fato pode ser explicado pelo sobrecarga física decorrente da dupla jornada de trabalho dessas profissionais, caracterizada pelo desenvolvimento de atividades nas escolas e com as obrigações dos afazeres domésticos, que podem contribuir para o surgimento da sintomatologia osteomuscular. Corroborando o que foi exposto anteriormente, um estudo realizado com 794 professores no município de Vitória da Conquista/BA verificou uma associação estatística significativa $(\mathrm{p}=0,0001)$ de alta sobrecarga doméstica com o sexo feminino (26).

Outro importante aspecto que também pode explicar a maior prevalência do referido quadro entre as professoras, é o fato da existência de diferença entre a massa muscular, a composição corporal e estatura das mulheres quando comparadas aos indivíduos do sexo masculino. Dessa forma, o sexo feminino se constitui num importante fator de risco para a presença de sintomas como a dor (27).

Os achados obtidos a partir da análise multivariada dos dados dos professores do município de Natal/RN, estão de acordo com os resultados demonstrados em 
outros estudos $(15,24,26,28)$, onde a o sexo feminino e a prática inadequada de atividade física também estavam associados à presença dos sintomas osteomusculares.

$\mathrm{Na}$ investigação realizada com os professores da rede pública de ensino de uma cidade do Estado de São Paulo, 46,5 \% dos participantes não praticavam atividades físicas (11). Outra pesquisa demonstrou que trabalhadores que praticavam exercícios físicos apresentavam níveis de severidade de sintomas menor quando comparados com aqueles que não os realizavam (16).

Fatores encontrados na realidade dos docentes investigados no presente estudo, como a alta carga horária de trabalho em sala de aula, o que somado ao progressivo aumento de atividades extra-classe, dentre elas as domésticas, além de questões financeiras referentes aos baixos salários recebidos por esses profissionais, podem ajudar no entendimento da não realização de atividades físicas de forma satisfatória.

Os professores vêm sendo progressivamente acometidos por diversas patologias, ignorando suas próprias necessidades de saúde, assumindo em muitas situações uma postura desanimadora e conformista, não investindo ou não tendo condições para reverter tal quadro (12).

É importante serem consideradas as limitações dos resultados provenientes da utilização de questionários auto-administráveis e de estudos transversais, os quais produzem uma imagem que retratam uma situação de saúde característica de um momento especifico de tempo, avaliando apenas os trabalhadores «sadios» e excluindo aqueles que por ventura estavam afastados das atividades laborais no momento da coleta por motivo de problemas de saúde.

De uma forma geral, foi observado que os professores da rede municipal de ensino da cidade de Natal/RN apresentaram alta prevalência de sintomas osteomusculares no decorrer de um período de um ano, apresentando o referido quadro uma associação estatisticamente significativa com o sexo feminino e a prática inadequada de atividade física.

Os resultados encontrados no estudo chamam atenção para o possível impacto que a sintomatologia osteomuscular pode ter na qualidade de vida desses indivíduos. São necessários maiores investimentos em pesquisas sobre a saúde dos professores, além da implantação de políticas públicas que tenha como objetivo principal a promoção de saúde para esse grupo de trabalhadores. 
É de fundamental importância a implantação de medidas para evitarem o agravamento do quadro exposto pelos resultados do presente estudo, o que caso aconteça poderá levar ao afastamento das atividades de trabalho de diversos professores, implicando em gastos com tratamentos de saúde e com questões previdenciárias, fazendo com que uma importante parte dos gastos públicos seja destinada a essas finalidades, ao invés de proporcionarem investimentos reais nas melhorias das condições de trabalho e saúde desse grupo de trabalhadores

Agradecimentos. Agradecemos a FAPESB (Fundação de Amparo à Pesquisa do Estado da Bahia) pelo auxílio referente à bolsa de apoio ao curso de doutoramento de Marcos Henrique Fernandes.

\section{REFERÊNCIAS}

1. Libâneo JC. Adeus Professor, Adeus Professora? Novas exigências educacionais e profissão docente. 9a Ed. São Paulo: Cortez; 2006.

2. Oliveira DA. A reestruturação do trabalho docente: precarização e flexibilização. Educ.Soc. 2004; 25(89): 1127-1144.

3. Gasparini SM, Barreto SM, Assunção AA. O professor, as condições de trabalho e os efeitos sobre sua saúde. Educação e Pesquisa. 2005; 31(2): 189-199.

4. Picoloto D, Silveira E. Prevalência de sintomas osteomusculares e fatores associados em trabalhadores de uma indústria metalúrgica de Canoas-RS. Cienc Saúde Coletiva. 2008; 13(2): 507-16.

5. Walsh IAP, Corral S, Franco RN, Canetti EEF, Alem MER, Coury HJCG Capacidade para o trabalho em indivíduos com lesões músculo-esqueléticas crônicas. Rev Saúde Pública. 2004; 38(2): $149-56$.

6. Brasil. Instituto Brasileiro de Geografia e Estatística. Síntese de Indicadores Sociais. 2004; p 15.

7. Barros ENC, Alexandre NMC. Cross-cultural adaptation of Nordic musculoskeletal questionnaire. Int Nurs Ver. 2003; 50(2): 101-08.

8. Brasil. Ministério da Saúde. Doenças relacionadas ao trabalho: manual de procedimentos para os senviços de saúde. Brasília, DF; 2001.

9. Souza KR, Santos MBM, Pina JÁ, Maria ABV, Carmo MAT, Jensen M. Atrajetória do Sindicato Estadual dos Profissionais da Educação do Rio de Janeiro (Sepe-RJ) na luta pela saúde no trabalho. Cienc Saúde Coletiva. 2003;8(4): 1057-68.

10. Borges LH. As Lesões por Esforços Repetitivos (LER) como índice do mal-estar no mundo do trabalho. Rev CIPA. 2000; 252: 50-61.

11. Barros, ME, Zorzal DC, Almeida FS, Iglesias RZ, Abreu VGV. Saúde e trabalho docente: a escola como produtora de novas formas de vida. Trabalho, Educação e Saúde. 2007; 5(1): 105123.

12. Carvalho AJFP, Alexandre NMC. Sintomas osteomusculares em professores do ensino fundamental. Rev. Bra. Fisioter. 2006; 10(1): 35-41.

13. Penteado RZ, Pereira IMTB. Qualidade de vida e saúde vocal de professores. Rev Saúde Pública. 2007; 41(2):236-43.

14. Nahas MV, Barros MVG, Francalacci VL. O pentáculo do bem estar: base conceitual para avaliação do estilo de vida dos indivíduos ou grupos. Rev. Bras. de Atividade e Saúde. 2000;5(2): 4859.

15. Hernandez JAE, Neto FXV, Oliveira TC, Rodrigues AA, Neto CHE, Voser RC. Validação de construto do instrumento perfil do estilo de vida individual. Rev. Eletrônica da Escola de Educação Física e Desportos - UFRJ. 2007; 3(1): 3-17. 
16. Bicudo-Pereira IMT, Penteado RZ, Bydlowski CR, Elmor MRD, Grazzelli ME. Escolas Promotoras de Saúde: onde está o trabalhador professor?. Saúde em Revista. 2003; 5(11): 29-34.

17. Pinheiro FA, Tróccoli BT, Carvalho CV. Validação do Questionário Nórdico de Sintomas Osteomusculares como medida de morbidade. Rev Saúde Pública. 2002; 36(3): 30712.

18. Marchiori F, Barros MEB, Oliveira SP. Atividade de trabalho e saúde dos professores: o programa de formação como estratégias de intervenção nas escolas. Trabalho, Educação e Saúde. 2005; 3(1): 143-170.

19. Neto AMS, Araújo TM, Dutra FRD, Azi GR, Alves RL, Kavalkievicz C, et al. Condições de trabalho e saúde de professores da rede particular de ensino de Salvador, Bahia. Rev. Baiana de Saúde Pública. 2000; 24(1/2): 42-56.

20. Delcor NS, Araújo TM, Reis EJFB, Porto LA, Carvalho FM, Silva MO, et al. Condições de trabalho e saúde dos professores da rede particular de ensino de Vitória da Conquista, Bahia, Brasil. Cad. Saúde Pública. 2004; 20(1): 187-196.

21. Codo W. Educação: carinho e trabalho. Petrópolis: Editora Vozes;1999.

22. Castro MLO. A educação na constituição de 1988 e a LDB: Lei de diretrizes e bases da educação nacional. Brasília: André Quicé;1998.p.278.

23. Carvalho AJFP, Alexandre NMC. Qualidade de vida e sintomas osteomusculares relacionados ao trabalho em professores do ensino fundamental. Fisioterapia Brasil. 2006; 7(4): 279284.

24. Porto LA, Reis IC, Andrade JM, Nascimento CR, Carvalho FM. Doenças ocupacionais em professores atendidos pelo centro de estudos da saúde do trabalhador (CESAT). Revista Baiana de Saúde Pública. 2004; 28(1): 33-49.

25. Brandão AG, Horta BL, Tomasi E. Sintomas de distúrbios osteomusculares em bancários de Pelotas e região: prevalência e fatores associados. Rev Bras Epidemiol. 2005; 8(3): 295-305.

26. Esteve JM. O mal-estar docente: A sala de aula e a saúde dos professores. Bauru-SP: EDUSC-Editora da Universidade do Sagrado Coração; 1999.

27. Araújo TM, Godinho TM, Reis EJFB, Almeida MMG. Diferença de gênero no trabalho docente e repercussões sobre a saúde. Ciência \& Saúde Coletiva. 2006; 11(4): 1117-1129.

28. Maciel ACC, Fernandes MB, Medeiros LS. Prevalência e fatores associados à sintomatologia dolorosa entre profissionais da industria têxtil. Rev Bras Epidemiolog. 2006; 9(1): 94102. 